\title{
Existential/Humanistic Treatment Modalities for Counseling Returning Combat Veterans
}

\author{
Guigno $C^{*}$, Miranda W and Hallmark C \\ Mental Health and Human Services Program, City University of New York, Kingsborough Community College, New York, USA
}

\begin{abstract}
Veterans suffering from psychological disorders that stem from combat is a problem that has not been appropriately or effectively addressed in the mental health professions. Posttraumatic Stress Disorder and Depression have consistently plagued returning combat veterans, and though mental health counseling is offered, current treatment modalities limit the scope of the therapeutic process. Mental Health Professionals have focused on Cognitive Behavioral Therapy and medication as sufficient treatment. This article suggests that while this may be a useful treatment modality for addressing symptoms it does not adequately help veterans to resolve the full trauma of combat.
\end{abstract}

Keywords: Returning combat veterans; Humanistic treatment

\section{Introduction}

In the United States of America, returning combat veterans (RCVs) are faced with the challenges of coming home from war zones where they experienced and/or witnessed violence and destruction. For many RCVs these experiences can be traumatic and create a significant degree of stress and in many cases depression. Typically, the most common diagnoses for RCVs who have been injured or have witnessed a fellow in arms getting injured or killed is Post Traumatic Stress Disorder (PTSD) and Major Depression (MD). According to the Rand Corporation, approximately $22 \%$ of RCVs exhibit symptoms of PTSD and depression [1]. Further, this study suggests that, despite the severe anxiety, fear and depression often associated with PTSD, $46 \%$ of RCVs prefer to seek psychological counseling outside of the Veterans Administration (VA) and $50 \%$ of those who did seek help within the VA report receiving "minimally adequate treatment." If left untreated, these symptoms may lead to serious and dangerous outcomes such as increased risk for substance abuse or suicide. In addition, according to a 2016 Veterans Administration report, $27 \%$ of RCVs who suffer from PTSD also experience signs of substance use disorder (SUD). It is important to point out that the authors of this report feel that $27 \%$ is a low representation of the actual numbers since not all veterans seek help through the VA. In addition, several recent studies have strongly suggested that the current limited quality of care and a significant absence of concern by the Mental Health community and the U.S government has also contributed to an increase in substance abuse, homelessness and suicide (according to the same VA report, 22 veterans commit suicide each day among the RCV population).

Over the last 50 years many RCVs who report experiencing symptoms of PTSD and depression also report that they feel neglected and in many cases ignored by the U.S. government. According to a report by the National Council for Behavioral Health, 30\% of returning veterans require mental health services and less than $50 \%$ of these actually receive these types of services. In addition, of those receiving treatment, only $30 \%$ receive treatment that is evidence based. It is this type of neglect, which ranges from the inadequate responses by governmental and institutional entities to the shortcomings of the mental health community, that serves to increase the RCV's feelings of numbness, being cut off from others/isolation, depression, shame and anger [2].

\section{Current Treatment Modalities}

The high rates of suicide and a lack of adequate treatment highlights the limitations that are inherent to the current treatment modality that is in place. Common Mental Health techniques used for treating PTSD include Cognitive Behavior Therapy (CBT), Exposure Therapy, Eye Movement Desensitization and Reprocessing (EMDR), and psychotropic medications such as anti-anxiety and antidepressants. These treatments are, for the most part, cost effective, short-term approaches that essentially teach the RCV to accommodate the trauma into their lives and focus on developing strategies that are tailored to reducing the symptoms of anxiety and depression associated with PTSD. These approaches devote little if any attention to helping the RCV confront the trauma that she/he experienced while in combat. While most MHPs would agree that CBT and the use of medication has had some success reducing symptoms of PTSD such as anxiety, the psychological impact of combat still remains unresolved. What is often not discussed during psychological counseling sessions is the psychological impact that trauma has on a RCV's sense of self in terms of how they view their sense of meaning and purpose.

Trauma has the ability to challenge the RCV's foundations of belief (meaning and purpose), Mental Health Professionals (MHPs) need to focus on developing treatment modalities that addresses this loss in meaning and purpose. This paper proposes the use of the Existential/ Humanistic approach for MHPs as an approach that will improve the quality of psychological care for RCVs. In implementing this type of modality, the impact of trauma can be addressed and a foundation for a healthy lifestyle can begin.

*Corresponding author: Charles Guigno, Director of the Mental Health \& Human Services Program, City University of New York, Kingsborough Community College, New York, USA, Tel: +1-917-714-8222; E-mail: Charles.Guigno@kbcc.cuny.edu

Received April 29, 2017; Accepted May 05, 2017; Published May 15, 2017

Citation: Guigno C, Miranda W, Hallmark C (2017) Existential/Humanistic Treatment Modalities for Counseling Returning Combat Veterans. J Ment Disord Treat 3: 139. doi:10.4172/2471-271X.1000139

Copyright: ( 2017 Guigno C, et al. This is an open-access article distributed unde the terms of the Creative Commons Attribution License, which permits unrestricted use, distribution, and reproduction in any medium, provided the original author and source are credited. 


\section{Limitations and Consequences of Current Treatment Modalities}

The current treatment modalities used for RCVs experiencing the effects of trauma are not effective. In addition to the RCV's high rate of suicide, there are other issues that impact the mental health of RCVs. Unfortunately, effective therapeutic attempts by trained MHPs to resolve the psychological impact of the traumatic experiences are often ignored. As a result, a significant number of RCVs report that they self-medicate in an attempt to mask the psychological effects of their traumatic combat experiences. According to the VA, alcohol and substance abuse is a great concern for RCVs, especially those with PTSD, depression, anxiety, or other severe mental illness [3].

The VA reports that $27 \%$ of veterans who have been diagnosed with PTSD also have a secondary diagnosis of substance use disorder (SUD) and several other studies have pointed out that 1 in 3 veterans seeking treatment for SUD also have been diagnosed with PTSD. Alcohol and the use of different types of substances becomes a coping mechanism, or a way to avoid facing the trauma that was experienced during combat. Altering states of consciousness or using chemicals to numb psychological pain is an active way of fostering the avoidance response that is seen in PTSD.

Many RCVs report that they are unable to control the painful and traumatic memories of combat. Further, they report that these memories can be overwhelming and as a result the RCV feels a need for immediate escape. Interestingly, many RCVs report that they are aware that alcohol and the use of other substances can be disruptive and make the symptoms of PTSD worse. However, significant numbers of RCVs report that they feel hopeless and helpless, and as a result, feel that self-medicating is their only choice.

The use of alcohol and substances can harm the individual's need for recovery and makes the recovery process more difficult. There is corroborating evidence of the relationship between the severity of the trauma experienced by veterans and their use of alcohol [4]. The more extreme the trauma experienced, the more alcohol use is reported. In addition, this study stated that there was a correlation between the amount of alcohol use and the perceived stigma of seeking mental health treatment. The authors also stated that the RCV's perception of reaching out and seeking psychological help is often viewed as a sign of weakness and would prefer, in many cases, to use alcohol or other substances (heroin, cocaine, marijuana) as their way to deal with the psychological distress that they are experiencing. Abusing alcohol and substances can have several destructive consequences such as, but not limited to, feelings of isolation, and feelings of detachment from family and friends.

When the trauma is not addressed effectively, the consequences of the mental and emotional anguish experienced can also lead to homelessness. There is a high rate of veterans who are homeless. Homeless veterans consist of $8.6 \%$ of the total homeless population in the nation (several studies suggested that a significant number of homeless RCVs are underreported or not reported at all). Mental illness, PTSD, and substance abuse are substantial contributors to homelessness in the RCV population, and the high number of veterans in the homeless population remains an issue [5].

\section{The Impact of Trauma for RCV}

The Diagnostic and Statistical Manual of Mental Disorders, 5th ed. has four diagnostic criteria that define a diagnosis of PTSD [6]. The first is intrusion symptoms, which are recurring involuntary distressing memories of the traumatic event. The second is avoiding potential stimuli associated with the traumatic event. The third is negative alterations in cognition and mood. This can include symptoms such as persistent negative emotional states and dissociative amnesia. The fourth is marked alterations in arousal to stimuli associated with the traumatic event. This is sometimes referred to as hyper-arousal. PTSD is highly comorbid with other mental disorders, such as depression, anxiety and substance use disorder. The APA also reports an association between PTSD and suicidal ideation and suicidal attempts.

Wounded or killed [7], while exposure to trauma is not rare to the human experience, the special types of trauma that RCVs have faced in Iraq and Afghanistan coupled with the prolonged psychological stress of being in a war zone, have led to almost half of those veterans experiencing posttraumatic stress symptoms [8].

The majority of RCVs enter the military at approximately 18 years of age. At this stage of life, adolescents are still forming a sense of selfworth [9]. In addition to developing an identity, adolescents often express feelings of invincibility, labeled the Invincibility Fable, where adolescents believe they are immune to common dangers, highlighting their proclivity for egocentrism [10]. While feeling invincible as a teenager may be a normal rite of passage, carrying that mindset into a combat zone places the adolescent at a higher risk of trauma, injury, or death. The notion of invincibility does not give the entering serviceman the mindset needed to experience combat and make lifesaving decisions. This issue is further exacerbated by the fact that any adolescent brain is not fully formed, and full development is not completed until early to late twenties [11]. Placing these adolescents in violent and unstable situations when they are not cognitively prepared may explain why an overwhelming number of RCVs come back from combat with severe symptoms of PTSD. By the time these servicemen have acclimated to their harsh military lifestyle, they are sent home to re-acclimate into a society they left with a drastically different perspective.

The experiences that come from a war zone can leave a deep impact on the psyche that can be very difficult for RCVs to overcome. The traumatic memories experienced in combat will often return pervasively and unexpectedly. These memories can be triggered by various external stimuli, such as loud noises or darkness. This leads to hyper-vigilance, the enhanced sense of awareness to surroundings adaptive in situations of threat, that doesn't allow for the RCV to enter a relaxed state when they are in social situations, or in crowded areas. These feelings can lead to isolation from others, including family and friends. The feelings of shame and embarrassment can also lead to deepening feelings of depression and anxiety that often accompany PTSD. There is a strong desire to suppress the trauma that was experienced. Alcohol or substance use can become a tool for actively suppressing the traumatic experiences and the feelings that come with them, while also creating additional problems for the RCV.

For many RCVs, combat experiences such as the death of comrades in arms force veterans to confront the idea that people who are "not supposed to die", die. It is this simple reality that challenges the RCV belief that friendships last forever. The death of those we love forces RCVs to resort to use defense mechanisms such as denial and suppression as a way of confronting the emotional pain of loss. In addition to coping with the emotional pain of a loved one, this loss also creates an atmosphere of mistrust for RCVs. RCVs begin to realize that they and others are not invincible and that the future is unpredictable.

When friends are lost in combat, RCVs develop a sense of learned 
helplessness. The feelings of helplessness will serve to deepen the effects of depression and anxiety [12]. While these feelings of isolation, guilt, and helplessness are disruptive throughout the RCVs life, there are ways to develop self-efficacy with the guidance of MHPs that can allow them to learn how to create meaning and purpose in their lives.

\section{Current Education and Training for Mental Health Professionals Counseling RCVs}

Traditionally, the goal of treatment when counseling RCVs is to minimize the anxiety and "inappropriate behavioral reactions" to stimuli that elicit memories of the combat experience. Based on recent data (suicide rates, rise in substances abuse rates and veteran rates of homelessness) these approaches (CBT and medication) are not in the best interests of RCVs. Although CBT has the potential to assist RCVs in changing learned behaviors to decrease effects of trauma, RCVs are typically offered a limited amount of counseling sessions, reducing the possibility of long-term recovery. Instead, medication is prescribed but not paired with regular counseling, which contradicts the recommendation that Selective Serotonin Reuptake Inhibitor (SSRI) drugs be accompanied by therapy. While medication can help RCVs with symptoms of trauma it is not a cure for PTSD or any other disorder resulting from combat. In fact, the overuse of medication can be counter-therapeutic because it implies that RCV's have a disease. This decreases the effectiveness of CBT and a number of other therapeutic modalities. Without consistent access to therapy, RCVs are not given the opportunity to build a foundation of trust with professionals which could lead to a feeling of understanding and support that they otherwise do not have. The authors of this article strongly believe that in order to help the RCV create a healthy quality of life, the MHP must first develop and gain an understanding and insight into the RCV's experience. In order to create change they must focus on helping the RCV to resolve their feelings of isolation, guilt, shame, helplessness and abandonment.

The first step in accomplishing this goal is to look at the RCV as a member of a unique group of individuals. This group, or culture, in the military have been neglected for a long time when tailoring treatment programs for military personnel. This culture is quite comprehensive, having its own set of norms, values, customs, and even its own language and class system, that must be recognized by MHPs [13]. In the military, presenting a flat affect, and showing no signs of weakness are valued traits [14]. Fear, vulnerability and focusing on "self " rather than the group are not valued or accepted by this culture. This culture, with all of its components must be considered by MHPs when dealing with RCVs who have adopted and developed these traits that are highly adaptive in a military environment, but may be counterproductive when facing the lingering effects of trauma and adjusting back to civilian life. The feelings of shame and embarrassment are difficult to bear in the context of military culture, which leads the RCV to be disappointed in himself/ herself. When an individual joins the military at a young age with the sense of strength and invulnerability, answering the call to service for a variety of reasons, a sense of pride and purpose follows. Violent combat however can have a significant impact on their sense of vulnerability. Witnessing the death of friend and physical injury on the field of battle exposes the RCV to experiences that they are not prepared for. Several RCVs report responding with fear and anxiety on their first day of deployment. One Marine interviewed by the authors spoke extensively about his shock and fear on his first day of battle when, for first time, it occurred to him that he could die today. His immediate response to his fear was that he was a failure and a disappointment to his family, friends, and country.
Young men and women join the military for a variety of different reasons. Some include patriotism, finding a sense of belonging, family tradition, or looking for a better quality of life. According to Abraham Maslow all individuals have an inherent desire to find a sense of acceptance and belonging [15]. For many, joining the military was a vehicle to fulfill this motivation. The military emphasizes the group mentality rather than the individual. Being a part of a team encourages the RCV to not only think about themselves but the group. While this can be beneficial in combat situations, confronted by military personnel in an overseas deployment or in the daily operations of military life, it can become a hurdle for the MHP when the RCV still has a sense of loyalty to military comrades and admitting to weakness puts the feeling of being inadequate for the group comes front and center. This mentality serves to encourage a distrust towards outsiders, an issue that the MHP must strive to overcome when working with RCVs.

Treatment modalities such as CBT use therapeutic techniques created to help clients identify and change negative views they have of themselves, others, and the world [16]. CBT has the potential to be a successful mode of therapy for RCVs if they are provided with the option of long term counseling. However, the typical amount of time allotted to RCV counseling is 3 to 6 months. Though a 6-month time frame may work for civilian CBT patients, RCVs are coping with experiences that far exceed the trauma that other patients seek treatment for. In order to effectively treat RCVs, MHPs must be given an appropriate amount of time to create the therapeutic environment and then build towards addressing the significant traumas that lead the RCV to seek help. It is with this long term mindset that methods such as CBT can create positive change in the challenges of PTSD and other combat issues.

\section{Existential Model as an Effective Treatment Modality}

An effective treatment modality is one that adopts techniques and philosophies that are designed to encourage the client to create a healthy quality of life. The Existential/Humanistic model, like many other models, emphasizes the importance of several of these techniques and strategies with a particular focus on the importance of developing a sense of meaning and purpose in people's lives.

\section{Attending}

The first step toward meaningful change within the therapeutic process is to establish therapeutic rapport between the MHP and the RCV that is based on mutual trust and respect. However, many RCV's report that trust and respect are issues that create anxiety, apprehension and a desire to withdraw and detach physically and emotionally. It therefore appears imperative that before any therapeutic rapport can be achieved, MHP's must create an environment where the RCV feels safe and accepted. Attending strategies are a useful and relatively easy technique for creating the foundation for safe and accepting atmosphere [17].

Attending strategies can be non-verbal and verbal. Simple nonverbal gestures such as eye contact and relaxed body posture (eg. Open arms, do not cross legs) will communicate to the RCV that the MHP is open and receptive to their feeling and thoughts. Verbal responses such as "tell me more " or "I know talking about this is hard, but please tell me more. I want to try and understand "communicates that the MHP is engaged and interested in learning more about the RCV's experiences and feelings. MHPs are reminded (initially) to refrain from using terms such as "I understand" or "I'm sure that was difficult." RCVs tend to hear these types of responses as disingenuous. RCVs report feeling 
insulted and wanting to detach from people who have no desire to be genuine and pretend that they understand their experiences. Finally, before any meaningful conversation regarding the psychological impact of combat can occur, the MHP needs to create an atmosphere in which the RCV feels that they have the MHP's undivided attention. It is important for RCVs to know that they have the freedom to discuss and express their feelings without fear of criticism or judgment.

\section{Establishing Therapeutic Rapport}

First and foremost, this therapeutic rapport must focus on the mutual trust and respect between the RCV and the MHP. This will take a period of time to achieve given the level of mistrust and uncertainty RCVs have adopted as a result of their combat trauma. One useful technique for creating mutual trust and respect is for the MHP and the RCV to discuss confidentiality. The concept and rules of confidentiality often serve to strengthen the therapeutic bond between MHP and $\mathrm{RCV}$. Once confidentiality has been established, the RCV will view the MHP as someone who values trust and respects the RCV as a person and as a partner in the therapeutic process.

After the discussion of confidentiality, the MHP is now ready to begin the initial stages of the helping process. Despite that fact that some degree of trust and respect has been established, it is usually beneficial for MHPs to use closed ended questions as a way to strengthen the bond between MHP and RCV. By asking questions such as "what is your favorite sport? "or" What type of music do you enjoy listening to?" presents the MHP as an interested and compassionate person who wants to get to know the RCV as a person and not just a "crazy" combat veteran. The MHP's neutral responses to closed ended questions is also an opportunity for the MHP to present themselves as an accepting non-judgmental individual.

\section{Therapeutic Rapport/Empathic Listening}

Once the MHP is viewed as a person who respects the RCV and accepts every aspect of their life without blame or judgment the MHP can now begin to explore the RCV's thoughts and feelings regarding their experiences. Asking open ended questions such as "How did it make you feel when....?" gives the MHP a unique opportunity to not only listen to but also empathize with the RCV's emotional reactions to their traumatic experiences. In addition to hearing what the RCV has to say, empathic listening also includes validating the RCV's feelings, and perhaps most importantly, it gives the MHP the opportunity to feel the RCV's sorrow, confusion and in many cases shame and guilt.

In addition to exploring feelings and validating the RCV's emotional pain and frustration, empathic listening also offers an opportunity to strengthen the therapeutic rapport. The MHP can utilize empathic listening as an opportunity to recognize and reinforce positive characteristics and values of the RCV. For example, RCVs are often confused and surprised to hear that seeking help is a sign of strength and courage. RCVs also find it difficult to accept and understand how others will value and love them despite their fear. The MHP needs to reinforce that it is the RCV's right to be accepted unconditionally by others [18]. As the MHP creates a relationship based on what Carl Rogers called unconditional positive regard, the MHP and RCV will together create an open and genuine relationship based on mutual trust and respect.

\section{Clarification/Modeling}

Once the MHP has established an open and genuine relationship based on mutual trust and respect, the MHP is now ready to take a more proactive approach with the RCV. It is important for the MHP to understand that initially many RCV may have some misperceptions regarding several important ideas, feelings and beliefs (such as men must be strong, and crying is a sign of weakness), and would benefit from discussing their confusion. MHPs are in a unique position to clarify many of these misperceptions. For example, MHPs need to clarify for RCV s that feelings such as fear and anxiety are psychologically appropriate (normal). RCVs need to understand that fear is not a sign of weakness and that anxiety and vulnerability are not issues that should not be repressed. MHP need to help the RCV understand the importance of exploring and taking steps toward resolving these feelings in a safe and confidential setting

MHPs need to create a setting where they can clarify and teach RCVs how to challenge and confront fear, anxiety and sadness. Further, MHP can take this opportunity to clarify the importance of resolving the issues that contribute to the RCV's feelings of detachment and isolation.

MHPs need to appreciate and understand that clarification can be a difficult and complicated task. Many RCVs will be reluctant to accept a new perspective out of fear that they will be betraying a code of ethics that they have sworn to defend. RCVs have been indoctrinated by military and community (family and friends) to believe that a person's worth and value is based on his/her strengths and fearless approach to adversity. To help change this perspective, MHPs serve as models/ mentors. As models, MHPs can reinforce, through their actions, that it is acceptable and appropriate for an adult to experience fear and that all people are in fact vulnerable. MHPs can also introduce healthy behaviors and attributes that contribute to a person's worth and value to themselves, to their families and to their community.

\section{Creating Meaning and Purpose}

Perhaps the most significant yet difficult aspect of the therapeutic experience is helping the RCV design and create a sense of meaning and purpose in their lives. This is a particularly difficult task for many RCVs because for so much of their lives they have been told what to do, how to think and how to feel. They have been taught that pleasing an authority figure brings rewards of acceptance, love and approval. Failure at this task creates feelings of inadequacy and failure.

For the most part individuals join the military looking to find and fulfill a sense of meaning and purpose in life. They are willing to fight for "what is right and moral", and because most of them feel invincible to any type of physical or emotional pain they clearly are not psychologically prepared for the trauma that they have experienced/ witnessed. Many are not prepared to accept the death of friends, the physical pain that they witnessed on the battlefield and certainly not the guilt (survivor guilt) and shame many of them feel when they return home.

In addition to the psychological impact of combat, many RCVs also feel alone and detached from people and the community that was once their support network. Family and friends that they once shared love and intimacy with now seem like strangers. Under these circumstances it is easy to understand why a RCV would be reluctant, or at the very least, find it difficult to engage in conversation that they may believe challenges and even attracts their fundamental beliefs. It is important for MHPs to understand that the goal of existential/humanistic therapy is not to change a person's fundamental beliefs but rather to help them design and create a lifestyle that complements their values and morals.

It is important for MHP and RCVs to understand that the criteria 
for creating a sense of meaning and purpose in life is not to please others in an attempt to feel accepted or successful, but rather to create a lifestyle and set of behaviors based on understanding of our values and morals.

Many RCVs report that when they returned home they felt confusion and a sense of betrayal. All ideas and values that they were led to believe to be true were now being questioned. Many RCVs report that this confusion was accompanied by fear and anxiety and that they were encouraged by others (military, MHPs, family and friends) to believe something is wrong with them and that they should repress and forget all painful memories and feelings. As a result, RCVs are made to feel worthless and ashamed. Under these circumstances it is easy to understand how a RCV would resist sharing their thoughts and feeling with a MHP.

\section{Conclusion}

From an Existential/Humanistic perspective, the role of the MHP in terms of helping the RCV to find meaning and purpose is not to be "the expert" who will cure the patient. Rather the MHP needs to be viewed as a therapeutic partner who is there to guide the RCV through the therapeutic process. The Existential/Humanistic MHP recognizes the RCV as an individual with a sense of honor, an individual who is loyal to others and most of all an individual with a desire to live their lives as kind, compassionate and honest individuals who want nothing more than to enjoy a genuine/authentic relationship with others. MHP need to help the RCV to understand that their purpose is not to fix the RCV. The Existential/Humanistic therapeutic process is about helping the RCV re-discover their core values and then guiding them and helping them design a lifestyle based on these values that they decide is meaningful and genuine. Specifically, the responsibility of the MHP is to help the RCV identify the values and characteristics that mark the Existential/Humanistic model, such as compassion, authenticity, and sensitivity. Secondly, to nurture and help the RCV incorporate these values which are the cornerstones for connecting with others and forming healthy relationships, based on mutual trust, mutual respect, and a commitment to personal growth.

Finally, it is important to note that the Existential/Humanistic model is not meant to replace CBT and medication. Rather, the intention of helping RCVs resolve the impact of combat trauma, and finding meaning and purpose, should be looked at as a vital addition to the already established treatment modality. This would create a more complete and powerful treatment plan for RCVs who are in need of a more comprehensive therapeutic experience. However, even though the Existential/Humanistic approach makes for a more comprehensive treatment modality, several RCVs have reported to the authors that despite an MHP's caring, professional, and sincere approach, a disconnect between the RCV and the MHP often occurs. It is this therapeutic gap that interferes, and at best requires more time, with establishing a therapeutic report based on mutual trust and respect. An effective strategy for addressing this issue is to train and educate interested RCVs as psychological peer counselors (mentors/models). This type of peer counseling has proven to nurture and strengthen the establishment of a therapeutic bond between RCV and MHP [19]. RCVs share a unique bond that cannot be replicated by MHPs who have not experienced combat. While the utility of MHPs is necessary to guide them to finding meaning and purpose, the relationship between RCVs and MHPs would be strengthened with the shared experiences of combat. This particular mindset, which is acquired with all the cultural aspects inherent to the military, if also understood by the MHP, would bridge a critical therapeutic gap which may take an MHP without military background much longer to overcome. If more RCVs were trained as MHPs, the potential for increased effectiveness of the Existential/Humanistic approach would have the power to greatly improve the lives of RCVs.

\section{References}

1. Hepner KA, Sloss EM, Roth CP, Krull H, Paddock SM, et al. (2016) Quality of care for PTSD and depression in the military health system.

2. National Council for Behavioral Health (2012) Meeting the behavioral needs of veterans.

3. U.S. Department of Veterans Affairs (2017) PTSD: National Center for PTSD.

4. Miller SM, Pedersen ER, Marshall GN (2017) Combat Experience and Problem Drinking in Veterans: Exploring the Roles of PTSD, Coping Motives, and Perceived Stigma. Addictive Behaviors, 66: 90-95.

5. National Alliance to End Homelessness (2015) Veteran Homelessness.

6. American Psychiatric Association (2013) Diagnostic and statistical manual of mental disorders (5thedn), Arlington, VA: American Psychiatric Publishing.

7. Xue C, Ge Y, Tang B, Liu Y, Kang P, et al. (2015) A meta-analysis of risk factors for combat-related PTSD among military personnel and veterans.

8. Morin R (2011) For many injured veterans, a lifetime of consequences. Pew Research Center.

9. Ciccarelli SK, White JN (2010) Psychology, an explanation. Upper Saddle River, New Jersey: Pearson Education Inc.

10. Berger KS (2009) The developing person through childhood and adolescence. New York, New York: Worth Publishers.

11. Johnson SB, Blum RW, Giedd JN (2009) Adolescent maturity and the brain The promise and pitfalls of neuroscience research in adolescent health policy. Journal of Adolescent Health 45: 216-221.

12. Maier SF, Seligman ME (1976) Learned helplessness: Theory and evidence Journal of Experimental Psychology 105: 3-46.

13. Hall LK (2011) The importance of understanding military culture. Social Work in Health Care 50: 4-18.

14. Williams MB, Poijula S (2013) The PTSD workbook. Oakland, CA: New Harbinger Publications, Inc.

15. Maslow AH (1987) Motivation and personality (3rdedn), McReynolds C (ed.) New York, NY: Harper and Row.

16. Shulman L (2009) The Skills of Helping Individuals, Families, Groups, and Communities. Belmont, CA: Brooks/Cole, Cengage Learning.

17. Cormier WH, Cormier LS (1991) Interviewing strategies for helpers: Fundamental skill and cognitive behavioral interventions. Pacific Grove: Brooks/Cole.

18. Rogers CR (1951) Client-centered therapy. Boston, MA: Houghton-Mifflin.

19. Church TE (2009) Returning veterans on campus with war related injuries and the long road back home. Journal of Postsecondary Education and Disability 22: 43-52. 